\title{
Depressive Symptoms in Alzheimer's Disease: Natural Course and Temporal Relation to Function and Cognitive Status
}

\author{
Roee Holtzer, PhD, ${ }^{* \dagger}$ Nikolaos Scarmeas, MD, ${ }^{*}$ Domonick J. Wegesin, PhD, ${ }^{*}$ Marilyn Albert, PhD, ${ }^{\ddagger}$ \\ Jason Brandt, PhD, ${ }^{\ddagger}$ Bruno Dubois, MD, ${ }^{\mathcal{S}}$ George M. Hadjigeorgiou, MD," and Yaakov Stern, PhD*
}

OBJECTIVES: To examine the natural course of depressive symptoms in patients with probable Alzheimer's disease (AD), specifically, the temporal relationship between depressive symptoms, function, and cognitive status.

DESIGN: Multicenter cohort study with follow-up of up to 14 years.

SETTING: Patients from the two Multicenter Study of Predictors of Disease Course in Alzheimer's Disease (Predictors Study) cohorts were recruited at five sites in the United States and Europe.

PARTICIPANTS: Patients diagnosed with probable $\mathrm{AD}$ $(\mathrm{n}=536)$ enrolled in a longitudinal study (Predictors Study). MEASUREMENTS: Depressive symptoms were evaluated at 6-month intervals using the Columbia Scale for Psychopathology in Alzheimer's Disease. The Modified MiniMental State (3MS) and Blessed Dementia Rating Scale (BDRS) were used to assess cognitive status and functional activity, respectively.

RESULTS: The prevalence of depressive symptoms was stable over the first 3 years of follow-up, at approximately $40 \%$. There was a significant drop to $28 \%$ and $24 \%$ in the fourth and fifth years of follow-up, respectively. Time-dependent Cox analysis revealed that functional activity (BDRS) but not cognitive status (3MS) was a significant predictor of the first episode of depressive symptoms during follow-up. Generalized estimating equation analyses showed that $\mathrm{AD}$ duration and functional activity but not cognitive status were significantly related to depressive symptoms over the entire follow-up period.

From the ${ }^{*}$ Cognitive Neuroscience Division, Taub Institute, G.H. Gertrude Sergievsky Center and Department of Neurology, College of Physicians and Surgeons, Columbia University Medical Center, New York, New York; ${ }^{\dagger}$ Ferkauf Graduate School of Psychology and Department of Neurology, Albert Einstein College of Medicine, Yeshiva University, Bronx, New York; ${ }^{\ddagger}$ Department of Psychiatry and Behavioral Sciences, Johns Hopkins University, Baltimore, Maryland; ${ }^{\S}$ Hôpital de la Salpêtrière, Paris, France; and "Department of Neurology, University of Thessaly, Larissa, Greece.

This work was supported in part by National Institute on Aging Grant AG07370 and National Institutes of Health Grant RR00645.

Address correspondence to Dr. Yaakov Stern, Cognitive Neuroscience Division, Taub Institute, G.H. Gertrude Sergievsky Center and Department of Neurology, College of Physicians and Surgeons, Columbia University Medical Center, New York, NY 10032. E-mail: ys11@columbia.edu

DOI: $10.1111 / \mathrm{j} .1532-5415.2005 .00535 . \mathrm{x}$
CONCLUSION: Depressive symptoms are common in $\mathrm{AD}$, but their prevalence decreases over time. Examination of the temporal relationship between depressive symptoms and risk factors suggests that decline in function but not in cognition precedes the first episode of depressive symptoms in patients with probable AD. J Am Geriatr Soc 53:2083-2089, 2005.

Key words: depressive symptoms function; cognition; Alzheimer's disease

$\mathrm{D}$ epression is common in Alzheimer's disease (AD). Estimates of the prevalence of depressive symptoms cluster around $30 \%$ to $50 \%$, although some estimates are as low as $1 \% .^{1}$ This variability is likely attributable to several factors, including different methods by which depression was diagnosed, ${ }^{2-4}$ differences in study populations, ${ }^{5-8}$ and whether the information was elicited from patients or from significant others. ${ }^{9}$

Several studies have demonstrated that depressive symptoms are persistent in $\mathrm{AD},{ }^{10-12}$ tend to occur early in the disease, ${ }^{13-15}$ and decrease over time, ${ }^{16}$ but reliable risk factors for depression, beyond previous episodes, have yet to be identified in prospective research. This may be, at least in part, attributed to the limited number of published prospective studies that were designed to assess this matter directly.

If depression in $\mathrm{AD}$ is uniquely related to disease course, then the relationship between depression and disease-severity indices, such as functional activity and cognition, should be examined. Although the relationship between cognitive status and psychopathology has been established, ${ }^{10,17}$ the association between cognition and depressive symptoms in $\mathrm{AD}$ remains equivocal. Studies have suggested that depressive symptoms are related to lesssevere cognitive dysfunction ${ }^{13,18}$ as well as to more-severe cognitive dysfunction, ${ }^{10,19,20}$ but several studies have found that depressive symptomatology in $\mathrm{AD}$ is not related to cognitive status. ${ }^{11,12,21-24}$ A cross-sectional study using a large cohort suggested that depression was related to higher cognitive status but lower functional activity. ${ }^{25}$ Finally, there appears to be a consensus that lower functional ac- 
tivity at baseline is related to depression, ${ }^{12,26-28}$ although the temporal relationship between depressive symptoms and risk factors such as functional activity and cognition has yet to be determined.

The present study was designed to characterize the natural course of depressive symptoms in a large sample of patients diagnosed with probable $\mathrm{AD}$ who were followed prospectively for up to 14 years. Furthermore, whether functional activity, cognitive status, and disease duration were important predictors of depressive symptoms in $\mathrm{AD}$ was examined. Two separate matters were addressed. First, the temporal relationship between disease severity indices and depressive symptoms was determined by examining whether decline in function and cognition predicted the first episode of depressive symptoms during the follow-up. Second, whether the relationship between these disease-severity indices and the depressive symptoms was generalized over the entire follow-up period was examined. To the authors' knowledge, this is the first study that provides such an analysis of prospective data for patients with $\mathrm{AD}$ followed from mild to severe stages of the disease.

Information regarding the natural course and risk factors of depression in $\mathrm{AD}$ is of significant value to clinicians and caregivers of demented patients. There are several expert recommendations for the treatment of depression in $\mathrm{AD},{ }^{29}$ but strong support for specific treatment recommendations is absent (for review, see ${ }^{1}$ ). Hence, knowledge regarding the natural course of depression and its relation to disease duration and severity may also assist in formulating specific treatment recommendations.

\section{METHODS}

\section{Participants}

A total of 536 patients enrolled in the Multicenter Study of Predictors of Disease Course in Alzheimer's Disease (Predictors Study) participated in the investigation. All patients were diagnosed with probable AD. ${ }^{30,31}$ Patients from two Predictors Study cohorts who were recruited at five separate sites participated in the present study. In the United States, patients from both cohorts were recruited at three sites: Columbia Medical Center $(\mathrm{n}=205)$, Johns Hopkins School of Medicine ( $\mathrm{n}=142)$, and Massachusetts General Hospital $(\mathrm{n}=117)$. In the second cohort, two additional sites were added: Hôpital de la Salpêtrière at Paris, France $(\mathrm{n}=38)$, and the University of Thessaly at Larissa, Greece $(\mathrm{n}=34)$. The inclusion/exclusion criteria and evaluation procedures of the Predictors Study have been fully described elsewhere. ${ }^{32,33}$ With respect to psychiatric history, exclusion criteria included a history of schizophrenia or schizoaffective disorder, electroconvulsive therapy within the preceding 2 years or a history of 10 or more electroconvulsive treatments in any one course, alcohol or drug dependence during the preceding 5 years, primary affective disorder within 1 year before onset of dementia, and evidence of other causes of dementia. At the Columbia site, patients were recruited from the Memory Disorder Center and from doctors' private offices through the Alzheimer's Disease Research Center. Each consecutive patient who met the inclusion/exclusion criteria of the study was included, with the exception of those who did not consent to participate or who lived too far away and were unable to return to the hospital for regular follow-up. At the Johns Hopkins site, patients were recruited from the Dementia Research Center and from private physicians. For the Massachusetts General Hospital site, patients were recruited from the Geriatric Neurobehavioral Center, an outpatient service. Patients at Hôpital de la Salpêtrière at Paris and at the University of Thessaly at Larissa were recruited from outpatient clinics as well.

In the Predictors Study, patients are evaluated every 6 months. The mean number of semiannual visits \pm standard deviation for the entire sample was $5.6 \pm 5.5$, with a maximum of 28 visits, indicating that some of the patients had been followed for up to 14 years. Attrition is low in the Predictors Study. Follow-up was complete for $94.5 \%$ of the two cohorts; only $5.5 \%$ had missing follow-up information for the period of the last year before the most updated data entry. The total number of patients followed each year from the initial evaluation through the fifth year was 536, 460, $335,243,171$, and 130 , respectively. Of the 406 patients who did not have the complete 5-year follow-up, 248 had died. The remaining 158 patients were mostly subjects from the second cohort at the U.S. and European sites who were enrolled in the Predictors Study but whose follow-up was shorter than 5 years. Patients' cognitive status, functional competence, and depression were evaluated at entry to the study and at 6-month intervals thereafter. Given the transient nature of depressive symptoms in $\mathrm{AD}$, it is possible that not all depressive episodes (i.e., depression that was shorter than 6 months) were captured in the analyses reported in the present investigation. The percentage of patients who were on antidepressant medications each year from the initial evaluation through the fifth year was $19 \%$, $21 \%, 20 \%, 18 \%, 24 \%$, and $21 \%$, respectively.

\section{Measures \\ Depressive symptoms}

Depressive symptoms were assessed using the Columbia University Scale for Psychopathology in Alzheimer's Disease (CUSPAD)..$^{20}$ The CUSPAD is a short, semistructured rating scale that clinicians or research technicians can administer to an informant. The frame of inquiry is the month before examination. Most items are scored dichotomously (i.e., symptom is present or absent). Psychometric properties of this instrument have been reported. ${ }^{20}$ Interrater reliability for the major symptom categories was established for concurrent rating of a single interview $(\kappa=0.74-1.00)$ and for separate interviews $(\kappa=0.53-0.73)$. Specifically with respect to depression, interrater reliability was consistent for separate $(\kappa=0.73)$ and concurrent $(\kappa=0.80)$ rating of a single interview. A trained research technician administered the CUSPAD to an informant at the initial evaluation and at subsequent 6-month intervals. For the purpose of the present study, three indices of depressive symptoms were used. The first dichotomous index, which was exclusive of physical symptoms of depression, determined whether the patient was sad. The second index used a five-level scale to determine the extent and frequency of depression exclusive of physical symptoms. The third continuous index included physical symptoms (sleep and appetite) that are often but not necessarily associated with depression. Although the validity of the CUSPAD has been 
established, this instrument is not designed to diagnose or discriminate between different depressive disorders per the Diagnostic and Statistical Manual of Mental Disorders, Fourth Edition, Text Revision, classification system.

\section{Cognition}

Cognition was assessed using the modified version of the Mini-Mental State Examination (3MS). ${ }^{34,35}$ The 3MS was administered in English at the U.S. sites and in French and Greek translated versions in Paris and Larissa, respectively. U.S. presidents, recall, and general knowledge items were replaced with suitable French and Greek figures in the French and Greek samples. Modifications to the original 3MS include the addition of Digit Span Forward and Backward, ${ }^{36}$ two additional calculation items, recall of the current and four previous presidents of the United States, confrontation naming of 10 items from the Boston Naming Test, ${ }^{37}$ one additional sentence to repeat, and one additional figure to copy. The $3 \mathrm{MS}$ has a maximum of 57 points, with lower scores indicating poorer cognitive function.

\section{Functional Dependence}

Functional activity was assessed using the Blessed Dementia Rating Scale (BDRS) Parts I and II. ${ }^{38}$ The range is between 0 and 17 , with higher scores indicating greater functional dependence.

\section{General Health Status}

Health status was calculated using the modified Charlson Comorbidity Index. ${ }^{39}$ The following conditions were evaluated: peripheral vascular status, chronic renal disease, systemic malignancy, hypertension, diabetes mellitus, congestive heart failure, myocardial infarction, chronic obstructive pulmonary disease, chronic liver disease, and abnormality of the abdomen and joints.

\section{Statistical Analyses \\ Descriptive Statistics}

Descriptive information, including the number of patients followed, demographic characteristics, general health (modified Charlson Comorbidity Index ${ }^{39}$ ), number of 6month visits, 3MS, BDRS, and depression evaluations, were tabulated. The percentage of patients per year experiencing depressive symptoms was reported for a 5 -year period and delineated in relation to yearly mean 3MS and BDRS scores.

\section{Time-Dependent Cox Analyses}

Time-dependent Cox analysis was used to evaluate risk factors that predicted the first episode of depressive symptoms during the follow-up period. Duration (in 6-month blocks) between the initial visit and development of the outcome or last evaluation without the outcome served as the timing variable in each model. Cognitive status (3MS) and functional activity (BDRS) served as time-dependent covariates. Age, sex, education, cohort, antidepressant medications use (at any point during the follow-up), and modified Charlson Comorbidity Index ${ }^{39}$ served as fixed covariates. The dichotomous index of depressive symptoms (exclusive of physical symptoms) from the CUSPAD served as the dependent measure. The time-dependent Cox analysis was used to accomplish two related objectives: determine whether changes over time in function and cognition predicted the first episode of depressive symptoms during the follow-up, and establish the directionality of the relationship between the time-dependent covariates and depression. To address this second objective, a secondary analysis was performed on a subsample of patients who had no history of depression before the follow-up and who were not depressed at the baseline evaluation.

\section{General Estimating Equations}

General estimating equations (GEEs) were used to evaluate the relationship between the risk factors and depression using the entire sample $(\mathrm{n}=536)$ over all available patient visits. This statistical method takes into account the multiple visits per patient as well as the likelihood that an individual's characteristics correlate with each other over time. The repeated measures per patient (up to 28 6-month visits) are treated as a cluster. A second advantage of GEE is that it takes into account the status or changing value of each covariate at each visit. Separate GEE models were run for the three depression indices. Cognitive status (3MS), functional activity (BDRS), and disease duration (time of follow-up) served as predictors. Analyses controlled for time, age, education, sex, antidepressant medication use, Charlson Comorbidity Index, and cohort.

\section{RESULTS}

Sample characteristics at baseline $(\mathrm{N}=536)$ are summarized in Table 1.

Of the 536 participants, $59 \%$ were female, and the majority were white. Mean age was $74.0 \pm 8.7$; mean education was $13.0 \pm 4.1$ years, respectively. Mean baseline $3 \mathrm{MS}$ was $38.2 \pm 6.1$, indicating an overall mild level of cognitive impairment; mean baseline BDRS score was $3.7 \pm 2.4$, indicating mild level of functional impairments. ${ }^{40}$

Table 1. Demographic Characteristics of the Sample $(\mathrm{N}=536)$ at Baseline and Follow-Up Information

\begin{tabular}{lcc}
\hline \multicolumn{1}{c}{ Characteristic } & Value & $\mathrm{N}$ \\
\hline Age, mean \pm SD & $74 \pm 8.7$ & \\
Education, years, mean \pm SD & $13 \pm 4.1$ & \\
3MS score, mean \pm SD & $38.2 \pm 6.1$ & \\
Blessed score, mean \pm SD & $37.5 \pm 23.5$ & \\
Charlson index, mean \pm SD & $0.48 \pm 0.82$ & \\
Illness duration, years, mean \pm SD* & $4.06 \pm 2.30$ & \\
Female, \% & 59 & \\
Depressed, \% & 39.2 & \\
Cohort 1, \% & 45 & \\
6-month follow-up visits, mean \pm SD & $5.6 \pm 5.5$ & 4,142 \\
Depression evaluations, mean \pm SD & $5.43 \pm 5.4$ & 3,557 \\
Function (Blessed) evaluations, & $5.45 \pm 5.4$ & 3,592 \\
$\quad$ mean \pm SD & & \\
Cognitive (3MS) evaluations, & $4.0 \pm 3.9$ & 2,940 \\
$\quad$ mean \pm SD & & \\
\end{tabular}

* Duration of illness from onset to baseline visit per neurologist's estimate. $\mathrm{SD}=$ standard deviation; 3MS = modified Mini-Mental State Examination . 


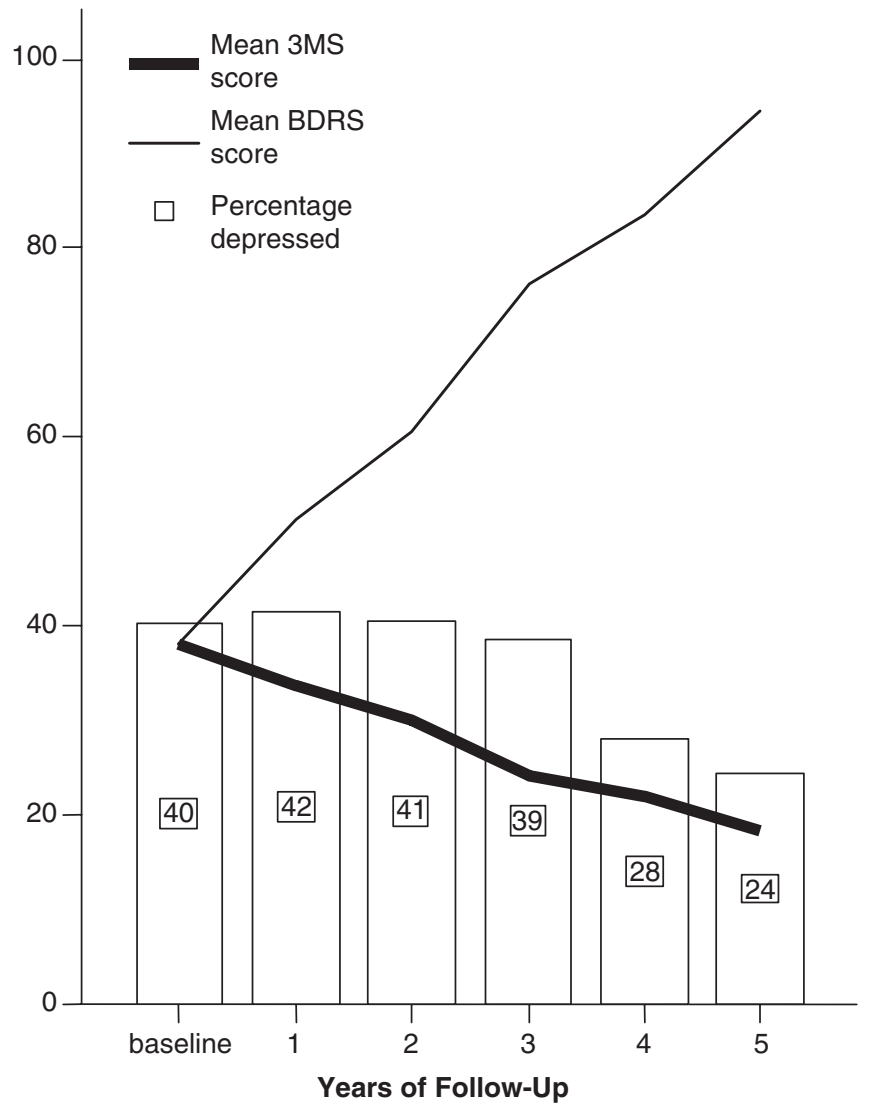

Figure 1. Percentage of patients deemed depressed at baseline through the fifth year of follow-up in relation to yearly mean modified Mini-Mental State Examination (3MS) and Blessed Dementia Rating Scale (BDRS) total scores.

\section{Descriptive Statistics}

The percentage of depressed patients in each of the 5 years of follow-up is presented in Figure 1. These findings are presented in relation to yearly mean $3 \mathrm{MS}$ and BDRS scores.

Figure 1 reveals that depressive symptoms were common and relatively stable during the first 3 years of the follow-up, ranging from $40 \%$ to $42 \%$ of the sample. A decrease in the prevalence of depressive symptoms to $28 \%$ and $24 \%$ of the sample was observed in the fourth and fifth years of follow-up, respectively. As expected, yearly mean 3MS scores decreased over time, indicating reduced cognitive function. BDRS scores increased during the follow-up, indicating a decline in functional activity. Prevalence rate of depressive symptoms in a subset of patients who had completed 5 years of follow-up $(n=130)$ was similar. The percentage of patients in this subsample deemed depressed each year in the initial evaluation through the fifth year was $39 \%, 43 \%, 42 \%, 40 \%, 30 \%$, and $29 \%$, respectively. Hence, it is unlikely that attrition biased the results. Some (but statistically insignificant, as judged using chi-square analysis) variability at baseline was noted between the different sites in the prevalence rates of depressive symptoms: Columbia (44\%), Johns Hopkins (36\%), Massachusetts General Hospital (44\%), Hôpital de la Salpêtrière (21\%), and University of Thessaly (33\%).

\section{Time-Dependent Cox Analysis}

Time-dependent Cox analysis examined risk factors of the first episode of depressive symptoms during the follow-up period using $3 \mathrm{MS}$ and BDRS as the time-dependent covariates. The results revealed that lower functional activity (BDRS) but not cognitive status (3MS) was a significant predictor of depression. Table 2 summarizes the results of the time-dependent Cox analysis.

A second time-dependent Cox analysis was performed on a subset of patients who reported no history of psychopathology before their enrollment in the Predictors Study and who were not depressed at baseline $(n=235)$. This secondary analysis was designed to provide evidence with respect to the directionality of the relationship between the time-dependent covariates and first episode of depressive symptoms. Consistent with the previous analysis, the results showed that decline in function but not cognition predicted the first episode of depressive symptoms during the followup period (Table 2, Model 2).

\section{GEE Analyses}

Separate GEE analyses were performed on three CUSPAD depression indices, with $3 \mathrm{MS}$, BDRS, and time serving as the predictors. Analyses controlled for time, age, sex, education, cohort, heath status, and antidepressant medi-

Table 2. Time-Dependent Cox Analyses: Prediction of First Episode of Depression

Model 1*

Model $2^{\dagger}$

Variable

Risk Ratio (95\% Confidence Interval) $P$-value

\begin{tabular}{|c|c|c|c|c|c|c|}
\hline Sex & 1.07 & $(0.86-1.32)$ & .56 & 1.17 & $(0.80-1.73)$ & .42 \\
\hline Age & 0.99 & $(0.98-1.00)$ & .10 & 0.98 & $(0.96-1.01)$ & .12 \\
\hline Education & 0.98 & $(0.95-1.01)$ & .12 & 1.02 & $(0.96-1.08)$ & .50 \\
\hline Cohort & 0.95 & $(0.76-1.18)$ & .62 & 1.08 & $(0.71-1.64)$ & .71 \\
\hline Charlson index & 1.05 & $(0.95-1.16)$ & .34 & 1.17 & $(0.79-1.72)$ & .43 \\
\hline Modified Mini-Mental State Examination & 1.01 & $(1.00-1.02)$ & .13 & 1.01 & $(0.99-1.04)$ & .26 \\
\hline
\end{tabular}

* Entire sample.

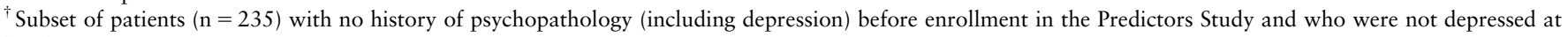
baseline.

\$Time-dependent covariates. 
Table 3. General Estimating Equation (GEE) Analyses: Prediction of Three Separate Depression Indices Across All Available Visits

\begin{tabular}{|c|c|c|c|c|c|c|c|c|c|}
\hline \multirow[b]{2}{*}{ Variable } & \multicolumn{3}{|c|}{ Model $1^{*}$} & \multicolumn{3}{|c|}{ Model $2^{\dagger}$} & \multicolumn{3}{|c|}{ Model $3^{\ddagger}$} \\
\hline & & & & $\beta(95 \% \mathrm{Cc}$ & onfidence Interval) & $P$-value & & & \\
\hline Sex & 0.002 & $(-0.061-0.066)$ & .95 & -0.038 & $(-0.167-0.092)$ & .57 & -0.167 & $(-0.413-0.080)$ & .19 \\
\hline Age & -0.003 & $(-0.007-0.001)$ & .14 & 0.000 & $(-0.008-0.008)$ & .97 & -0.016 & $(-0.031-0.001)$ & .03 \\
\hline Education & 0.000 & $(-0.009-0.008)$ & .95 & -0.005 & $(-0.022-0.012)$ & .56 & -0.028 & $(-0.062-0.005)$ & .10 \\
\hline $\begin{array}{l}\text { Antidepressant } \\
\text { medications }\end{array}$ & 0.049 & $(-0.015-0.113)$ & .14 & 0.127 & $(-0.016-0.271)$ & .08 & -0.205 & $(-0.057-0.466)$ & .13 \\
\hline Charlson index & 0.016 & $(-0.019-0.042)$ & .46 & -0.010 & $(-0.049-0.069)$ & .74 & -0.056 & $(-0.055-0.167)$ & .32 \\
\hline Cohort & -0.015 & $(-0.083-0.052)$ & .66 & -0.062 & $(-0.200-0.075)$ & .37 & -0.167 & $(-0.109-0.442)$ & .24 \\
\hline Time & -0.022 & $(-0.037-0.007)$ & .004 & -0.064 & $(-0.092-0.037)$ & $<.001$ & -0.220 & $(-0.276-0.156)$ & $<.001$ \\
\hline $\begin{array}{l}\text { Modified Mini-Mental } \\
\text { State Examination }\end{array}$ & 0.003 & $(0.000-0.006)$ & .03 & -0.005 & $(-0.001-0.011)$ & .11 & -0.007 & $(-0.005-0.019)$ & .26 \\
\hline $\begin{array}{l}\text { Blessed Dementia } \\
\text { Rating Scale }\end{array}$ & 0.002 & $(0.001-0.002)$ & $<.001$ & -0.003 & $(0.001-0.005)$ & $<.001$ & 0.013 & $(0.009-0.017)$ & $<.001$ \\
\hline
\end{tabular}

* Depression is dichotomous exclusive of physical symptoms.

${ }^{\dagger}$ Frequency of depression exclusive of physical symptoms.

${ }^{\ddagger}$ Total depression scores inclusive of physical symptoms.

cation use. Results of the analyses are summarized in Table 3.

Consistent with the descriptive data, time was negatively associated with depression in all three models. (The prevalence of depression decreased over time.) BDRS scores were significant predictors of depression in all three models, indicating that decline in functional activity was related to increased depression. In contrast, 3MS scores were not significant predictors of depression in two of the three models. The relationship between 3MS and depression was statistically significant in Model 1 . These findings are consistent with the time-dependent Cox analysis demonstrating that, although decline in functional activity was related to depression, cognitive function was not a reliable predictor of depressive symptoms in $\mathrm{AD}$.

\section{DISCUSSION}

The present study examined the natural course and prevalence of depressive symptoms in a large cohort of patients diagnosed with probable $\mathrm{AD}$, followed longitudinally for up to 14 years. Risk factors for depression were identified. Specifically, whether functional activity, cognitive status, and time were related to the risk of experiencing depressive symptoms was evaluated.

The prevalence of depressive symptoms was stable at approximately $40 \%$ during the first 3 years of follow-up, with a significant drop in the fourth and fifth years of follow-up. These findings are consistent with previous studies indicating that depression is common in the early phase of the disease ${ }^{13-15}$ and then decreases over time. ${ }^{16}$ These results may explain some of the reported variability of depressive symptoms in $\mathrm{AD}$, because the prevalence rates varied substantially as a function of time and disease course. It should be emphasized that changes in prevalence of depression over the course of follow-up are not likely to reflect fluctuations in the number of patients evaluated each year. Indeed, such prevalence rates were similar in a subset of patients who were assessed at every 6-month evaluation during the 5-year follow-up. Furthermore, the negative effect of time on depression, which was evident in all three GEE models, substantiated the descriptive data in demonstrating that the prevalence of depression declined over time. The possibility cannot be excluded that compromised communication in the later phases of $\mathrm{AD}^{41}$ may have limited the ability to assess depression, but given the variability in communication skills observed in the advanced stages of the disease, ${ }^{42}$ it is unlikely that the drop in prevalence of depressive symptoms in the fourth and fifth years of followup can be attributed exclusively to patients' inability to communicate that they were depressed.

The findings of this investigation extend previous research in supporting an association between function and depression in AD. ${ }^{12,25-28}$ Decline in function predicted the first episode of depressive symptoms during the follow-up period and was associated with greater risk of depression when taking into account all patient visits. This association remained reliable even after controlling for the effects of a large number of variables that could have attenuated the relationship between depression and functional activity.

Moreover, the present study was designed to evaluate the temporal relationship between functional activity and depression. Lower functional activity may precede or follow the onset of depressive symptoms. The time-dependent Cox analysis provided the most convincing evidence in support of the former interpretation. Changes over time in functional activity served as a time-dependent covariate, which predicted the first episode of depressive symptoms during the follow-up period. The direction of this relationship was further demonstrated in a subset of patients who reported no history of psychopathology (inclusive of depression) before being diagnosed with probable $\mathrm{AD}$ and who were not depressed at baseline. Hence, the possibility that premorbid history of psychopathology might confound these findings does not appear to be supported in this sample.

Determining the direction of the relationship between functional activity and depression is important inasmuch as it has implications for the etiology and treatment of 
depression in $\mathrm{AD}$. A few recent studies suggest that the combination of cognitive rehabilitation and pharmacological intervention may result in some improvement in cognitive status and functional activity ${ }^{43-45}$ in patients with $\mathrm{AD}$. In this context of rehabilitative treatment, one possible implication is that structuring the patient's environment to maximize independence or possibly even the perception of independence may help to reduce the extent of depressive symptoms.

In contrast to functional activity, cognitive function was not a significant predictor of first episode of depression during the follow-up, nor was it reliably related to depression when taking into account all available patient visits. Although the lack of association between cognitive function and depression in $\mathrm{AD}$ has been reported in other studies, ${ }^{21}$ two related indices of AD severity (3MS and BDRS) are differentially related to the risk of experiencing depression during the course of the disease. Moreover, a previous study revealed that subjective memory complaints were unrelated to depressive symptoms in preclinical AD. ${ }^{14}$ Taking these together, it appears that, whereas cognitive decline or the perception of reduced cognitive capacity are not related to depression, the practical consequences of having the disease, which results in loss of independence (i.e., function), are consistently related to increased risk of depression in AD.

A limitation of the present study is the assessment of depression. Although the CUSPAD has been validated for use in $\mathrm{AD},{ }^{20}$ it was developed primarily to examine phenomenological features. Consequently, partial response to treatment and fluctuation over time in symptom severity may not be detected with this instrument. Nonetheless, an attempt was made to address this limitation by deriving a second depression index that was sensitive to the frequency of depressive symptoms. A third index of depression inclusive of physical symptoms was derived as well. The relationship between depression and functional activity was demonstrated in all analyses in this paper irrespective of how depression was assessed. This suggests that the findings are likely not attributable to the method by which depression was evaluated. This study relied on informant reports to assess patients' depressive symptoms. Whereas patients tend to underreport symptoms, informant variables, such as perceived burden, may be related to greater reporting of depressive symptoms in patients. Hence, it cannot be excluded that informants' variables may have partially influenced the results as well. Another limitation is the assessment of the antidepressant medications. Although all analyses controlled for the effect of a large range of antidepressant medications, this study was not designed to monitor therapeutic aspects such as medication type and dosage and adherence to the prescribed treatment. Consequently, and despite the stringent control over the possible effect of antidepressant medications on the findings, it is possible that this study underestimated the effect of pharmacological intervention on depressive symptoms in AD.

The longitudinal design of this study has significant advantages over cross-sectional studies and prospective studies of short follow-up when evaluating the manifestation of depression over time and in relation to functional and cognitive status in AD. Specifically, when assessed longitudinally, changes in depressive symptoms can be attributed directly to disease progress and to the decline in functional activity. In contrast, findings from cross-sectional studies and short follow-up prospective studies cannot be used to make inferences regarding such disease processes. (For a recent review concerning the limitations of crosssectional studies with respect to disease processes see ${ }^{46}$.)

Further confidence in the finding of the present study is attributed to the careful diagnosis and follow-up that the patients received and the high confirmation rate of $\mathrm{AD}$ in autopsy cases. ${ }^{47,48}$ The assessment of functional activity, cognitive status, and depression relies on standardized measures that have been validated in patients with $\mathrm{AD}$ and on uniform application of widely accepted criteria in a consensus diagnostic conference procedure. The semiannual evaluations conducted throughout the follow-up period increase the diagnostic reliability and validity of emotional, cognitive, and functional status. Finally, because the participants in this study were recruited from two separate cohorts and five different centers in the United States and Europe, the findings reported in the investigation are likely to generalize to patients seen in memory disorder clinics and AD centers in hospitals. Generalizability to communitybased samples will have to be addressed in future research.

In summary, this study delineated the natural course of depression in $\mathrm{AD}$ and evaluated the possible relationship between three disease indices and depressive symptoms. Whereas functional activity and the time of follow-up were reliable predictors of depression, cognitive function was not. Furthermore, the findings suggest that decline in functional activity precedes the onset of depressive symptoms in AD. Future research should directly examine whether, in the context of rehabilitative treatment, increasing patients' level or perception of independence reduces depressive symptoms in $\mathrm{AD}$.

\section{ACKNOWLEDGMENTS}

Financial Disclosure: R. Holtzer, N. Scrameas, D. Wegesin, J. Brandt, M. Albert, B. Dubois, and M. Hadjigeorgiou indicate no financial disclosures. Y. Stern is a consultant for Eisai Pharmaceuticals and Cortex Pharmaceuticals.

Author Contributions: R. Holtzer, N. Scrameas, and Y. Stern: concept, design, analysis and interpretation of data, manuscript preparation. D. Wegesin, J. Brandt, M. Albert, B. Dubois, and G.M. Hadjigeorgiou: acquisition of subjects and data, manuscript preparation.

Sponsor's Role: None.

\section{REFERENCES}

1. Olin JT, Katz IR, Meyers BS et al. Provisional diagnostic criteria for depression of Alzheimer disease. Am J Geriatr Psychiatry 2002;10:129-141.

2. Regier DA. Limitations of diagnostic criteria and assessment instruments for mental disorders. Arch Gen Psychiatry 2000;57:109-115.

3. Eaton WW, Neufeld K, Chen LS et al. A comparison of self-report and clinical diagnostic interviews for depression. Arch Gen Psychiatry 2000;57:217-222.

4. Murphy JM, Monson RR, Laird N et al. A comparison of diagnostic interview for depression in the Stirling County Study. Arch Gen Psychiatry 2000;57:230236.

5. Harwood DG, Barker WW, Ownby RL. Depressive symptoms in Alzheimer's disease: An examination among community dwelling Cuban American patients. Am J Geriatr Psychiatry 2000;8:84-91.

6. Harwood DG, Barker WW, Ownby RL. Clinical characteristics of communitydwelling black Alzheimer's disease patients. J Natl Med Assoc 2005;92: 424-429.

7. Liu CY, Fu JL, Teng EL. Depressive disorders in Chinese patients with Alzheimer's disease. Acta Psychiatr Scand 1999;100:451-455. 
8. Skoog I. The prevalence of psychotic, depressive and anxiety syndromes in demented and non-demented 85-year-olds. Int J Geriatr Psychiatry 1993; 8:247-253.

9. Mackenzie TB, Robiner WN, Knopman DS. Differences between patient and family assessments of depression in Alzheimer's disease. Am J Psychiatry 1989; 146:1174-1178.

10. Devanand D, Jacobs DM, Tang MX et al. The course of psychopathology in mild to moderate Alzheimer's disease. Arch Gen Psychiatry 1997;54:257-263.

11. Levy ML, Cummings JL, Fairbanks LA et al. Longitudinal assessment of symptoms of depression, agitation, and psychosis in 181 patients with Alzheimer's disease. Am J Psychiatry 1996;153:1438-1443.

12. Garre-Olmo J, Lopez-Pousa S, Vilalta-Franch J et al. Evolution of depressive symptoms in Alzheimer's disease: One year follow-up. Alzheimer Dis Assoc Disord 2003;17:77-85.

13. Zubenko GS, Zubenko WN, McPherson S. A collaborative study of the emergence and clinical features of the major depressive syndrome in Alzheimer's disease. Am J Psychiatary 2003;160:857-866.

14. Berger AK, Fratiglioni I, Forsell Y et al. The occurrence of depressive symptoms in the preclinical phase of AD. A population-based study. Neurology 1999;53:1998-2002.

15. Chen P, Ganguli M, Mulsant BH et al. The temporal relationship between depressive symptoms and dementia. Arch Gen Psychiatry 1999;56:261-266.

16. Li YS, Meyer JS, Thornby J. Longitudinal follow-up of depressive symptoms among normal versus cognitively impaired elderly. Int J Geriatr Psychiatry 2001;16:718-727.

17. Holtzer R, Tang M-X, Devanand D et al. Psychopathological features in Alzheimer's disease: Course and relationship with cognitive status. J Am Geriatr Soc 2003;51:953-960.

18. Lopez OL, Boller F, Becker TL. Alzheimer's disease and depression: Neuropsychological impairment and progression of the illness. Am J Psychiatry 1990;147:855-860.

19. Burns A, Jacoby R, Levy R. Psychiatric phenomena in Alzheimer's disease. Br J Psychiatry 1990;157:72-76.

20. Devanand DP, Miller L, Richards $M$ et al. The Columbia University Scale for Psychopathology in Alzheimer's disease. Arch Neurol 1992;49:371-376.

21. Ballard C, Bannister C, Solis $\mathrm{M}$ et al. The prevalence, associations and symptoms of depression amongst dementia sufferers. J Affect Disord 1996;36:135-144.

22. Cooper JK, Mungas D, Weiler P. Relation of cognitive status and abnormal behaviors in Alzheimer's disease. J Am Geriatr Soc 1990;38:867-870.

23. Cummings JL, Miller B, Hill MA et al. Neuropsychiatric aspects of multiinfract dementia and dementia of the Alzheimer type. Arch Neurol 1987; 44:389-393.

24. Lazarus LW, Newton N, Cohler B et al. Frequency and presentation of depressive symptoms in patients with primary degenerative dementia. Am J Psychiatry 1987;144:41-45.

25. Payne JL, Lyketsos CG, Steele C et al. Relationship of cognitive and functional impairment to depressive features in Alzheimer's disease and other dementias. J Neuropsychiatry Clin Neurosci 1998;10:440-447.

26. Lyketsos CG, Steele C, Baker L et al. Major and minor depression in Alzheimer's disease: Prevalence and impact. J Neuropsychiatry Clin Neurosci 1997;9:556-561.

27. Pearson JL, Teri L, Reifler BV et al. Functional status and cognitive impairment in Alzheimer patients with and without depression. J Am Geriatr Soc 1989;37: 1117-1121.
28. Fits AG, Teri L. Depression cognition and functional ability in patients with Alzheimer's disease. J Am Geriatr Soc 1994;42:186-191.

29. Lyketsos CGI, Campo L, Steinberg M et al. Treating depression in Alzheimer disease: Efficacy and safety of sertraline therapy and the benefits of depression reduction: The DIADS. Arch Gen Psychiatry 2003;60:737-746.

30. McKhann G, Drachman D, Folstein M et al. Clinical diagnosis of Alzheimer's disease. Report of the NINCDS-ADRDA Work Group under the auspices of the Department of Health and Human Services Task Force on Alzheimer's disease. Neurology 1984;34:939-944.

31. Diagnostic and Statistical Manual of Mental Disorders, Revised, 3rd Ed. Washington, DC: American Psychiatric Press, Inc., 1987.

32. Stern Y, Albert SM, Sano M et al. Assessing patient dependence in Alzheimer's disease. J Gerontol 1994;49:M216-M222.

33. Richards M, Folstein M, Albert M et al. Multi-center study of predictors of disease course in Alzheimer's disease (the 'Predictors Study'). II. Baseline findings. Alzheimer Dis Assoc Disord 1993;7:22-32.

34. Stern Y, Sano M, Paulson J et al. Modified Mini-Mental State Examination. Validity and reliability. Neurology 1987;37:179.

35. Folstein MF, Folstein SE, McHugh PR. 'Mini-mental state'. A practical method for grading the cognitive state of patients for the clinician. J Psychiatr Res 1975;12:189-198.

36. Wechsler D. Wechsler Adult Intelligence Scale - Revised. New York: The Psychological Corporation, 1981.

37. Goodglass H, Kaplan E. The Assessment of Aphasia and Related Disorders, 2nd Ed. Philadelphia: Lea \& Febiger, 1983.

38. Blessed G, Tomlinson BE, Roth M. The association between quantitative measures of senile change in the cerebral grey matter of elderly subjects. $\mathrm{Br}$ J Psychol 1968;114:797-811.

39. Charlson ME, Pompei P, Ales KL et al. A new method of classifying prognostic comorbidity in longitudinal studies: Development and validation. J Chronic Dis $1987 ; 40: 373-383$.

40. Eastwood MR, Lautenschlaeger E, Corbin S. A comparison of clinical methods for assessing dementia. J Am Geriatr Soc 1983;31:342-347.

41. Ripich DN, Fritsch T, Ziol E et al. Compensatory strategies in picture description across severity levels in Alzheimer's disease: A longitudinal study. Am J Alzheimer Dis 2000;15:217-228.

42. Bayles KA, Tomoeda CK, Cruz RF et al. Communication abilities of individuals with late-stage Alzheimer disease. Alzheimer Dis Assoc Disord 2000; 14:176-181.

43. Loewenstein DA, Acevedo A, Czaja SJ et al. Cognitive rehabilitation of mildly impaired Alzheimer disease patients on cholinesterase inhibitors. Am J Geriatr Psychiatry 2004;12:395-402.

44. Rogers JC. Understanding Alzheimer disease: From diagnosis to rehabilitation. Phys Occup Ther Geriatr 2002;20:103-123.

45. Bottino C, Carvalho I, Alvarez AM et al. Cognitive rehabilitation in Alz heimer's patients: Multidisciplinary team report. Arq Neuropsiquiatr 2002; 60:70-79.

46. Kraemer HC, Yesavage JA, Taylor A et al. How can we learn about developmental processes from cross-sectional studies, or can we? Am J Psychiatry 2000;157:163-179.

47. Scarmeas N, Hadjigeorgiou GM, Papadimitriou A et al. Motor signs during the course of Alzheimer's disease. Neurology 2004;63:975-982.

48. Scarmeas N, Albert M, Brandt J et al. Motor signs predict poor outcomes in Alzheimer disease. Neurology 2005;64:1696-1703. 